PROCEEDINGS OF THE

AMERICAN MATHEMATICAL SOCIETY

Volume 140, Number 8, August 2012, Pages 2613-2625

S 0002-9939(2011)11114-6

Article electronically published on December 8, 2011

\title{
ON A CLASS OF POLYNOMIALS RELATED TO BARKER SEQUENCES
}

\author{
PETER BORWEIN, STEPHEN CHOI, AND JONAS JANKAUSKAS
}

(Communicated by Matthew A. Papanikolas)

AbStract. For an odd integer $n>0$, we introduce the class $\mathcal{L} P_{n}$ of Laurent polynomials

$$
P(z)=(n+1)+\sum_{\substack{k=1 \\ k \text { odd }}}^{n} c_{k}\left(z^{k}+z^{-k}\right)
$$

with all coefficients $c_{k}$ equal to -1 or 1 . Such polynomials arise in the study of Barker sequences of even length, i.e., integer sequences having minimal possible autocorrelations. We prove that polynomials $P \in \mathcal{L} P_{n}$ have large Mahler measures, namely, $M(P)>(n+1) / 2$. We conjecture that minimal Mahler measures in the class $\mathcal{L} P_{n}$ are attained by the polynomials $R_{n}(z)$ and $R_{n}(-z)$, where

$$
R_{n}(z)=(n+1)+\sum_{\substack{k=-n \\ k \text { odd }}}^{n} z^{k}
$$

is a polynomial with all the coefficients $c_{k}=1$. We prove that

$$
M\left(R_{n}\right)>n-\frac{2}{\pi} \log n+O(1) .
$$

The results of experimental computations on polynomials in the class $\mathcal{L} P_{n}$ suggest two conjectures which could shed light on the long-standing Barker problem.

\section{INTRODUCTION}

Let $a_{0}, a_{1}, \ldots, a_{n}$ be the sequence of complex numbers, all of modulus $\left|a_{j}\right|=1$. For this sequence, the aperiodic autocorrelation coefficients $c_{k},-n \leqslant k \leqslant n$, are defined by

$$
c_{k}:=\sum_{j=0}^{n-k} a_{j} \bar{a}_{k+j} \quad \text { for } \quad 0 \leqslant k \leqslant n \quad \text { and } \quad c_{-k}:=c_{k} .
$$

The sequences $a_{k}$ having small autocorrelation values $\left|c_{k}\right|$ have a long history in signal processing; see [1, 6], 7]. In particular, the Barker sequences are those which have all numbers $a_{k}$ equal to 1 or -1 and $\left|c_{k}\right| \leqslant 1$ for $k \neq 0$. It is widely

Received by the editors January 23, 2011 and, in revised form, March 6, 2011.

2010 Mathematics Subject Classification. Primary 11B83, 11C08, 30C10; Secondary 42A05, $94 \mathrm{~A} 05$

Key words and phrases. Laurent polynomials, Barker conjecture, Barker polynomials, Barker sequences, Littlewood polynomials, Mahler measures, $L_{p}$ norms, aperiodic autocorrelations.

The first and second authors are supported by NSERC, Canada.

A visit of the third author at IRMACS Center, Simon Fraser University, was funded by the Lithuanian Research Council (student research support project).

(C)2011 American Mathematical Society Reverts to public domain 28 years from publication 
believed that only finitely many Barker sequences exist. Turyn and Storer 18. proved that no Barker sequences of odd length exist for $n \geqslant 13$. Various restrictions on the possible values of $n$ were derived in papers [4, [5], 8], 12, [16. It is known that if a Barker sequence of even length $n>13$ exists, then either $n=$ 189260468001034441522766781604 , or $n>2 \cdot 10^{30}$ by the result of [9], though the nonexistence problem still remains unsolved and has been for more than 45 years. Kaltofen and Mossinghoff [2] introduced a polynomial version of the Barker conjecture in terms of irreducibility of polynomials. This is a theme we exploit further in this paper.

Recall that a polynomial $p(z) \in \mathbb{Z}[z]$ is called a Littlewood polynomial if all coefficients of $p(z)$ are equal to 1 or -1 . The set of Littlewood polynomials is denoted by

$$
\mathcal{L}_{n}:=\left\{p(z)=a_{0}+\cdots+a_{n} z^{n}: a_{j}=1 \text { or } a_{j}=-1,0 \leqslant j \leqslant n\right\} .
$$

The polynomial $p \in \mathcal{L}_{n}$ is called a Barker polynomial if the coefficients $a_{0}, a_{1}$, $\ldots, a_{n}$ form a Barker sequence of length $n+1$. Since the autocorrelations of the coefficients do not change in magnitude by replacing the polynomial $p(z)$ with $p(-z)$ or $-p(-z)$, one can normalize Barker polynomials using the conditions $a_{n}=a_{n-1}=$ 1. By the results of [18], there exist precisely five normalized Barker polynomials of even degree $n$, namely: $z^{2}+z-1, z^{4}+z^{3}+z^{2}-z+1, z^{6}+z^{5}+z^{4}-z^{3}-z^{2}+z-1$, $z^{10}+z^{9}+z^{8}-z^{7}-z^{6}-z^{5}+z^{4}-z^{3}-z^{2}+z-1$, and $z^{12}+z^{11}+z^{10}+z^{9}+z^{8}-$ $z^{7}-z^{6}+z^{5}+z^{4}-z^{3}+z^{2}-z+1$. The only known normalized Barker polynomials of odd degree $n$ are the three polynomials $z+1, z^{3}+z^{2}-z+1$ and $z^{3}+z^{2}+z-1$. It is believed that there are no more Barker polynomials of odd degree. In [2, it was shown that the nonexistence of Barker polynomials of even degree for $n>13$ would follow from the irreducibility of certain integer polynomials in $\mathbb{Z}[x]$. By using the results of Saffari [13] on the property of flatness, Borwein and Mossinghoff [3] proved that if an infinite sequence of Barker polynomials exists, they would have extremely large $L_{s}$ norms on the unit circle. This would give answers to the old conjectures of Littlewood [19, [20] and Newman [10, 11] for the class of Littlewood polynomials $p(z) \in \mathcal{L}_{n}$.

In the present paper we introduce and study a new class of polynomials associated to the Barker polynomials (with a focus on those of odd degree).

Definition 1.1. Let $n$ be an odd integer. Define the class $\mathcal{L} P_{n}$ as the class of polynomials of the form

$$
P(z)=(n+1)+\sum_{k=-n, k \text { odd }}^{n} c_{k} z^{k}
$$

with coefficients $c_{k}=-1$ or 1 and $c_{-k}=c_{k}$.

Barker polynomials $p(z) \in \mathcal{L}_{n}$ are related to the polynomials in $\mathcal{L} P_{n}$ in the following way: if $p(z)$ is a Barker polynomial of odd degree, then the Laurent polynomial defined by $P(z):=p(z) p(1 / z)$ belongs to the class $\mathcal{L} P_{n}$ as can be seen easily from the equations (1.1). It seems that the special form of the polynomials $P(z) \in \mathcal{L} P_{n}$ is natural in the investigations of Barker polynomials of odd degree. Our paper is just a starting point for these investigations. We shall concentrate our efforts in the two main directions. In the first place, we give the results on the $L_{s}$ norms and especially the Mahler measure of the polynomials in the class 
$\mathcal{L} P_{n}$. Secondly, we state some conjectures on the measures and the irreducibility of $P \in \mathcal{L} P_{n}$.

\section{Main Results}

We start by making some definitions. Recall that a function $P(z)$ is called a Laurent polynomial (centered at the origin) if it is a polynomial in $z$ and $1 / z$ with complex coefficients, $P(z) \in \mathbb{C}[z, 1 / z]$. For any real number $s \geq 1$, the $L_{s}$ norm of $P(z)$ on the unit circle $|z|=1$ is defined by

$$
\|P\|_{s}=\left(\frac{1}{2 \pi} \int_{0}^{2 \pi}\left|P\left(e^{i t}\right)\right|^{s} d t\right)^{1 / s} .
$$

If $P \neq 0$ is a Laurent polynomial, then $P(z)=z^{-m} Q(z)$ for some polynomial $Q \in \mathbb{C}[z], Q(0) \neq 0$ and some $m \in \mathbb{N}$ :

$$
Q(z):=c_{0}+c_{1} z^{2}+\cdots+c_{n} z^{n}=c_{n} \prod_{j=1}^{n}\left(z-\alpha_{j}\right) \in \mathbb{C}[z] .
$$

The Mahler measure of $P(z)$ and $Q(z)$ is defined by

$$
M(P):=M(Q)=\left|c_{n}\right| \prod_{j=1}^{n} \max \left\{1,\left|\alpha_{j}\right|\right\} .
$$

In view of Jensen's formula, one has

$$
\log M(P)=\frac{1}{2 \pi} \int_{0}^{2 \pi} \log \left|P\left(e^{i t}\right)\right| d t .
$$

In Proposition 2.1 we list some basic properties of the polynomials in the class $\mathcal{L} P_{n}$. Among all polynomials in $\mathcal{L} P_{n}$, polynomials with all coefficients $c_{k}$ equal to 1 or, alternatively, all coefficients $c_{k}=-1$ are of special interest. We define

$$
R_{n}(z):=(n+1)+\sum_{\substack{k=-n \\ k \text { odd }}}^{n} z^{k} .
$$

We will show that $R_{n}(z)$ and $R_{n}(-z)$ has several interesting extremal properties.

Proposition 2.1. Let $P \in \mathcal{L} P_{n}$. Then $P\left(e^{i t}\right)$ takes real nonnegative values for $t \in[0,2 \pi) . P(z)=0$ holds for some $z$ of modulus 1 if and only if $P(z)=R_{n}(z)$ and $z=-1$ or $P(z)=R_{n}(-z)$ and $z=1$. For each $P \in \mathcal{L} P_{n}$, one has

$$
\|P\|_{1}=n+1
$$

and

$$
\|P\|_{2}=\left((n+1)^{2}+(n+1)\right)^{1 / 2} .
$$

In addition, we also have

$$
\|P\|_{4} \leqslant\left\|R_{n}\right\|_{4}=\left((n+1)\left(3 n^{3}+29 n^{2}+49 n+24\right) / 3\right)^{1 / 4} .
$$

In Proposition 2.2 below we identify a more precise subclass of the polynomials in $\mathcal{L} P_{n}$, related to the products of Barker polynomials. We note that this is just a restatement of the theorem of Turyn and Storer [15], [18. The proof is also given in [3]. 
Proposition 2.2. Suppose that $p(z) \in \mathcal{L}_{n}$ is a Barker polynomial of odd degree $n$. Then $n=4 m^{2}-1$ for some $m \in \mathbb{Z}$ and the coefficients $c_{k}$ of the polynomial $P(z)=p(z) p(1 / z) \in \mathcal{L} P_{n}$ in the formula (1.1) satisfy $c_{n+1-k}=-c_{k}$ for $1 \leqslant k \leqslant n$, so that $P(z)$ can be written as

$$
P(z)=(n+1)+T(z)+T(1 / z)
$$

where the polynomial

$$
T(z):=c_{n} z^{n}+\cdots+c_{k} z^{k-1}+\cdots+c_{1} z
$$

is negative reciprocal: $z^{n+1} T(1 / z)=-T(z)$.

The result of Theorem 4.1 in the paper 3 is that the hypothetical Barker polynomials $p(z)$ of degree $n$ have Mahler measures $M(p)>\sqrt{n+1}-1$ for $n$ large enough. On the other hand, it is known that $M(p)<\|p\|_{1}<\sqrt{n+0.91}$ holds for all Littlewood polynomials of degree $n$, by Theorem 5.2 of [3], which was proven by refining the estimate of Newman. Therefore one possible approach for solving the nonexistence problem of Barker would be to show that the Mahler measure of any Barker polynomial would be too large to satisfy an upper bound, which we speculate is likely. However, completely new methods are needed to improve the earlier estimates, an observation made by Newman himself [10. In search of a new approach, we give the next result on the uniform lower bound for the Mahler measures of polynomials in $\mathcal{L} P_{n}$.

Theorem 2.3. Suppose that $P \in \mathcal{L} P_{n}$. Then we have

$$
M(P)>\frac{n+1}{2} .
$$

The constant $1 / 2$ in Theorem 2.3 does not seem to be optimal, and we hope to refine it in subsequent attempts. We conjecture that:

Conjecture 2.4. Among all the polynomials $P \in \mathcal{L} P_{n}$ the polynomials $R_{n}(z)$ and $R_{n}(-z)$ have minimal Mahler measures:

$$
\min _{P \in \mathcal{L} P_{n}} M(P)=M\left(R_{n}\right) .
$$

We have verified Conjecture 2.4 computationally up to degree $n=39$.

Conjecture 2.5. The Mahler measures of polynomials $R_{n}(z)$ satisfy

$$
M\left(R_{n}\right)=n-1+\delta_{n},
$$

where the error term $\delta_{n}<0.725$ for all $n$ sufficiently large and the sequence $\delta_{n}$ is slowly decreasing. It seems that $\delta_{n}$ is bounded from below, probably $\delta_{n}>0$. If this is true, then the monotone convergence theorem implies the existence of the limit $\lim _{n \rightarrow \infty}\left(M\left(R_{n}\right)-(n-1)\right)$.

This second conjecture has been verified for polynomials $R_{n}$ of degree $n<250$. If proven, these conjectures would lead to the nontrivial improvement of Theorem 4.1 of [3]: if $p(z)$ is a Barker polynomial, then $M(p)^{2}=M(p(z)(p(1 / z))$ for $p(z) p(1 / z) \in$ $\mathcal{L} P_{n}$ implies $M(p)>\sqrt{n-1}$ for $n$ sufficiency large. Note that the trivial upper bound $M(P)<\|P\|_{1}=n+1$ for all $P \in \mathcal{L} P_{n}$ follows from the monotonicity of $L$-norms and Proposition 2.1. We remark that the computation of the exact values of the Mahler measure of polynomials is quite difficult; see, for instance, a survey by C. Smyth [14. As a first step towards Conjecture 2.4 we prove the inequality for the Mahler measures of polynomials $R_{n}$ : 
Theorem 2.6. For the polynomials $R_{n}(z)$ defined above:

$$
M\left(R_{n}\right)>n-\frac{2}{\pi} \log n+O(1)
$$

as $n \rightarrow \infty$.

We would like to conclude this section with a conjecture on the reducibility of polynomials in the class $\mathcal{L} P_{n}$, which, combined with Proposition 2.2 implies the nonexistence of Barker polynomials of odd degree $n \geqslant 5$. This conjecture has been verified computationally for $n<75$. An analogous conjecture for Barker polynomials in the even degree case was introduced in [2].

Conjecture 2.7. For each $n \geqslant 5$, the subset of polynomials $P \in \mathcal{L} P_{n}$ with coefficients $c_{n+1-k}=-c_{k}$ for $1 \leqslant k \leqslant n$ in (1.1) contains only two elements which are reducible in $\mathbb{Z}[z, 1 / z]$ (up to the factor of the form $\pm z^{m}, m \in \mathbb{Z}$ ). These elements are $P(z)=R_{n}(z)$ and $P(z)=R_{n}(-z)$ defined by (2.2). The polynomial $R_{n}(z)$ factors into $(z-1)(1-1 / z) Q(z)$, where $Q \in \mathbb{Z}[z, 1 / z]$ is irreducible.

\section{LEMMAS}

For the proofs of Theorems 2.3 and 2.6. we need the following results.

Lemma 3.1. Let $k \in \mathbb{Z}$. Then we have

$$
\frac{1}{2 \pi} \int_{0}^{2 \pi} \log \left(4 \cos ^{2}(k t / 2)\right) d t=0
$$

and

$$
\frac{1}{2 \pi} \int_{0}^{2 \pi} \log \left(4 \sin ^{2}(k t / 2)\right) d t=0
$$

Proof. Consider $f(z)=\left(z^{k}+1\right)^{2}$. Then $\left|f\left(e^{i k t}\right)\right|=\left|e^{i k t}+1\right|^{2}=\left|e^{i k t / 2}+e^{-i k t / 2}\right|^{2}=$ $4 \cos ^{2}(k t / 2)$. By (2.1), the first integral is just $\log M\left(\left(z^{k}+1\right)^{2}\right)=0$. Similarly, the second integral is $\log M\left(\left(z^{k}-1\right)^{2}\right)=0$.

Lemma 3.2. Let $a, b, x$ and $\alpha$ be real numbers. Suppose $a+b \cos \alpha \geq 0$. Then we have

$$
a \sin x+b \sin (x+\alpha)=c \sin (x+\beta),
$$

where

$$
c=\sqrt{a^{2}+b^{2}+2 a b \cos \alpha}
$$

and

$$
\beta=\tan ^{-1}\left(\frac{b \sin \alpha}{a+b \cos \alpha}\right) .
$$

The proof is straightforward and is omitted.

Lemma 3.3. Let $n \in \mathbb{N}$ be odd and $I(a):=[a-1 / n, a+1 / n] \subseteq \mathbb{R}$ for $a \in \mathbb{R}$. Then the function

$$
h(t):=(n+1) \sin t+\sin (n+1) t
$$

satisfies the following:

$$
\log |h(t)|= \begin{cases}\log n+\log |t|+O(1), & \text { if } t \in I(0), \\ \log n+O(1 / n), & \text { if } t \in I(\pi / 2) \cup I(3 \pi / 2), \\ 3 \log n+3 \log |t-\pi|+O(1), & \text { if } t \in I(\pi) .\end{cases}
$$


Proof. Since $h(t)$ is $C^{\infty}(\mathbb{R})$, so $h(t)$ is well approximated by its Taylor expansion. Indeed, if we let $N:=n+1$, then the derivatives of $h(t)$ are

$$
h^{(k)}(t)=\left\{\begin{array}{lll}
(-1)^{\frac{k}{2}}\left(N \sin t+N^{k} \sin N t\right) & \text { if } k \equiv 0 & (\bmod 2), \\
(-1)^{\frac{k-1}{2}}\left(N \cos t+N^{k} \cos N t\right) & \text { if } k \equiv 1 & (\bmod 2) .
\end{array}\right.
$$

We also observe that $N$ is even and $\log N=\log n+O(1 / n)$.

We now suppose $t \in I(a)$.

Suppose first that $a=\pi / 2$ or $a=3 \pi / 2$. Then we have $h(a)= \pm N,\left|h^{\prime}(t)\right| \leqslant 2 N$, $|t-a| \leqslant 1 / n$ and the Taylor expansion of $h(t)$ about $a$ is

$$
h(t)= \pm N+h^{\prime}(\theta)(t-a)
$$

for some $\theta \in I(a)$. It follows that

$$
\log |h(t)|=\log N+\log \left(1+\frac{h^{\prime}(\theta)(t-a)}{N}\right)=\log n+O(1 / n) .
$$
have

For $a=0$, we have $h(0)=h^{\prime \prime}(0)=0, h^{\prime}(0)=2 N$ and $\left|h^{\prime \prime \prime}(t)\right|<2 N^{3}$. Hence we

$$
h(t)=2 N t+\frac{h^{\prime \prime \prime}(\theta) t^{3}}{6}=2 N t\left(1+\frac{h^{\prime \prime \prime}(\theta) t^{2}}{12 N}\right)
$$

for some $\theta \in I(0)$. Now since

$$
\left|\frac{h^{\prime \prime \prime}(\theta) t^{2}}{12 N}\right|<\frac{1}{6}\left(\frac{N}{n}\right)^{2}=\frac{1}{6}\left(1+\frac{1}{n}\right)^{2} \leq \frac{2}{3}
$$

so

$$
\frac{1}{3}<1-\left|\frac{h^{\prime \prime \prime}(\theta) t^{2}}{12 N}\right| \leq\left|1+\frac{h^{\prime \prime \prime}(\theta) t^{2}}{12 N}\right| \leq 1+\left|\frac{h^{\prime \prime \prime}(\theta) t^{2}}{12 N}\right|<\frac{5}{3}
$$

Hence

$$
\log |h(t)|=\log N+\log |t|+O(1) .
$$

Finally, for $a=\pi$, we have $h(a)=h^{\prime}(a)=h^{\prime \prime}(a)=h^{(4)}(a)=0, h^{\prime \prime \prime}(t)=N-N^{3}$ and $\left|h^{(5)}(t)\right|<2 N^{5}$. Hence we get

$h(t)=\frac{N-N^{3}}{3 !}(t-\pi)^{3}+\frac{h^{(5)}(\theta)}{5 !}(t-\pi)^{5}=\frac{N-N^{3}}{6}(t-\pi)^{3}\left(1+\frac{h^{(5)}(\theta)(t-\pi)^{2}}{20\left(N-N^{3}\right)}\right)$

for some $\theta \in I(\pi)$. Now since

$$
\left|\frac{h^{(5)}(\theta)(t-\pi)^{2}}{20\left(N-N^{3}\right)}\right|<\frac{N^{5}}{10\left(N^{3}-N\right) n^{2}}=\frac{1}{10}\left(\frac{(n+1)^{4}}{n^{3}(n+2)}\right)<\frac{1}{10}\left(1+\frac{1}{n}\right)^{3} \leq \frac{4}{5},
$$

so

$$
\frac{1}{5}<\left|1+\frac{h^{(5)}(\theta)(t-\pi)^{2}}{20\left(N-N^{3}\right)}\right|<\frac{9}{5}
$$

Hence

$$
\log |h(t)|=\log \left(N^{3}-N\right)+\log |t-\pi|^{3}+O(1)=3 \log n+3 \log |t-\pi|+O(1) .
$$

This completes the proof. 
Lemma 3.4. Let $n \geq 2$. If $a \in\{0, \pi\}$, then we have

$$
\int_{a-2 / n}^{a+2 / n} \log |\sin t| d t=-\frac{4}{n} \log n+O\left(\frac{1}{n}\right)
$$

and

$$
\int_{a-1 / n}^{a+1 / n} \log |\sin (t+\beta(n, t))| d t=-\frac{2}{n} \log n+O\left(\frac{1}{n}\right)
$$

where

$$
\beta(n, t)=\tan ^{-1}\left(\frac{\sin (n t)}{n+1+\cos (n t)}\right) .
$$

If $a \in\{\pi / 2,3 \pi / 2\}$, then we have

$$
\int_{a-2 / n}^{a+2 / n} \log |\sin t| d t \ll \frac{1}{n} \text { and } \int_{a-1 / n}^{a+1 / n} \log |\sin (t+\beta(n, t))| d t \ll \frac{1}{n^{2}} .
$$

Proof. If $a=\frac{\pi}{2}$ or $a=\frac{3 \pi}{2}$, then $0<\sin \left(\frac{\pi}{2}-1\right) \leqslant|\sin t| \leqslant 1$ in the interval $[a-2 / n, a+2 / n]$. Hence

$$
\left|\int_{a-2 / n}^{a+2 / n} \log \right| \sin t|d t| \leq\left(\frac{4}{n}\right)\left|\log \sin \left(\frac{\pi}{2}-1\right)\right| \ll \frac{1}{n} .
$$

If $a=0$ or $a=\pi$, then we write

$$
\log |\sin t|=\log \left|\frac{\sin (t-a)}{t-a}\right|+\log |t-a| .
$$

Note that the function $|\sin (t-a) /(t-a)|$ is continuous and strictly positive in $[a-2 / n, a+2 / n]$. Hence

$$
\int_{a-2 / n}^{a+2 / n} \log \left|\frac{\sin (t-a)}{t-a}\right| d t \ll \frac{1}{n}
$$

On the other hand,

$$
\int_{a-2 / n}^{a+2 / n} \log |t-a| d t=\frac{4}{n}\left(\log \frac{2}{n}-1\right)=-\frac{4}{n} \log n+O\left(\frac{1}{n}\right)
$$

and hence

$$
\int_{a-2 / n}^{a+2 / n} \log |\sin t| d t=-\frac{4}{n} \log n+O\left(\frac{1}{n}\right) .
$$

From Lemma 3.2 with $a=n+1, b=1, x=t$ and $\alpha=n t$, we have

$$
\sin (t+\beta(n, t))=\frac{(n+1) \sin t+\sin (n+1) t}{c(n, t)},
$$

where $c(n, t)=\sqrt{(n+1)^{2}+1+2(n+1) \cos (n t)}$. Let $h(t)=(n+1) \sin t+\sin (n+1) t$. By Lemma 3.3, if $a=\pi / 2$ or $3 \pi / 2$, then $\log |h(t)|=\log n+O(1 / n)$ for all $t \in$ $[a-1 / n, a+1 / n]$. Hence

$$
\int_{a-1 / n}^{a+1 / n} \log |h(t)| d t=2 \frac{\log n}{n}+O\left(1 / n^{2}\right)
$$

In addition, $\log c(n, t)=\log n+O(1 / n)$. Hence

$$
\int_{a-1 / n}^{a+1 / n} \log |\sin (t+\beta(n, t))| d t \ll \frac{1}{n^{2}} .
$$


If $a=0$, then $\log |h(t)|=\log n+\log |t|+O(1)$ for all $t \in[a-1 / n, a+1 / n]$ and hence

$$
\int_{a-1 / n}^{a+1 / n} \log |h(t)| d t=\frac{2 \log n}{n}+\int_{-1 / n}^{1 / n} \log |t| d t+O(1 / n)=O(1 / n) .
$$

If $a=\pi$, then $\log |h(t)|=3 \log n+3 \log |t-\pi|+O(1)$ for all $t \in[a-1 / n, a+1 / n]$ and hence

$$
\int_{a-1 / n}^{a+1 / n} \log |h(t)| d t=\frac{6 \log n}{n}+3 \int_{\pi-1 / n}^{\pi+1 / n} \log |t-\pi| d t+O(1 / n)=O(1 / n) .
$$

Hence

$$
\int_{a-1 / n}^{a+1 / n} \log |\sin (t+\beta(n, t))| d t=-\frac{2}{n} \log n+O\left(\frac{1}{n}\right) .
$$

\section{Proofs of the Propositions AND THEOREMS}

Proof of Proposition 2.1. Let $P \in \mathcal{L} P_{n}$. Then

$$
P(z)=(n+1)+\sum_{k=-n, k \text { odd }}^{n} c_{k} z^{k}=(n+1)+\sum_{k=1, k \text { odd }}^{n} c_{k}\left(z^{k}+z^{-k}\right) .
$$

For $z=e^{i t}, t \in[0,2 \pi)$,

$$
\begin{aligned}
P\left(e^{i t}\right) & =(n+1)+\sum_{\substack{k=1 \\
k \text { odd }}}^{n} c_{k}\left(e^{i k t}+e^{-i k t}\right) \\
& =(n+1)+\sum_{\substack{k=1 \\
k \text { odd }}}^{n} 2 c_{k} \cos k t \geqslant(n+1)-(n+1)=0,
\end{aligned}
$$

since $n$ is odd and $c_{k}= \pm 1$. The equality is possible if and only if all $c_{k} \cos (k t)=-1$. In particular, for $k=1, \cos t=1$ or $\cos t=-1$. In the first case, $t=0$; hence all the coefficients $c_{k}=-1$. In the second case $t=\pi$ and all $c_{k}=1$. This proves that $P\left(e^{i t}\right) \geqslant 0$ for all $P \in \mathcal{L} P_{n}$ and $P\left(e^{i t}\right)=0$ only if $P(z)=R_{n}( \pm z)$. Thus for any $P \in \mathcal{L} P_{n}$,

$$
\begin{aligned}
\|P\|_{1} & =\frac{1}{2 \pi} \int_{0}^{2 \pi}\left|P\left(e^{i t}\right)\right| d t \\
& =\frac{1}{2 \pi} \int_{0}^{2 \pi} P\left(e^{i t}\right) d t=(n+1)+\sum_{\substack{k=1 \\
k \text { odd }}}^{n} \frac{2 c_{k}}{2 \pi} \int_{0}^{2 \pi} \cos k t d t=(n+1) .
\end{aligned}
$$

By Parserval's formula,

$$
\|P\|_{2}^{2}=(n+1)^{2}+\sum_{\substack{k=1 \\ k \text { odd }}}^{n} 2 c_{k}^{2}=(n+1)^{2}+(n+1) .
$$

It remains to compute the $L_{4}$-norm. By Parserval's formula, $\|P\|_{4}^{4}$ is equal to the sum of squares of coefficients of the polynomial $P^{2}$. Clearly, if all $c_{k}=1$, then all 
the coefficients of $P^{2}$ are positive and achieve maximal values. Thus the maximal $L_{4}$-norm is achieved by the polynomial $R_{n}$. By direct calculation,

$$
\begin{aligned}
R_{n}(z)^{2} & =\left((n+1)+\sum_{k=-n, k \text { odd }}^{n} z^{k}\right)^{2} \\
& =(n+1)^{2}+2(n+1) \sum_{k=-n, k \text { odd }}^{n} z^{k}+\left(\sum_{k=-n, k \text { odd }}^{n} z^{k}\right)^{2} \\
& =(n+1)^{2}+2(n+1) \sum_{k=-n, k \text { odd }}^{n} z^{k}+\sum_{\substack{m=-2 n \\
m \text { even }}}^{2 n}\left(\sum_{\substack{k_{1}, k_{2}=-n, k_{i} \text { odd } \\
k_{1}+k_{2}=m}}^{n}\right) z^{m} \\
& =\sum_{m=-2 n}^{2 n} d_{m} z^{m},
\end{aligned}
$$

where

$$
d_{m}:= \begin{cases}2(n+1) & \text { if } m \text { is odd and }|m| \leq n, \\ 0 & \text { if } m \text { is odd and }|m|>n, \\ (n+1)(n+2) & \text { if } m=0, \\ n+1-\frac{|m|}{2} & \text { if } m \text { is even and } 0<|m| \leq 2 n .\end{cases}
$$

Therefore,

$$
\begin{aligned}
\left\|R_{n}(z)\right\|_{4}^{4} & =\sum_{m=-2 n}^{2 n} d_{m}^{2} \\
& =((n+1)(n+2))^{2}+(n+1)(2(n+1))^{2}+2 \sum_{m=1}^{n}(n+1-m)^{2} \\
& =\frac{1}{3}(n+1)\left(3 n^{3}+29 n^{2}+49 n+24\right) .
\end{aligned}
$$

Proof of Theorem 2.3. As in the proof of Proposition 2.1, for $z=e^{i t}, t \in[0,2 \pi)$,

$$
P\left(e^{i t}\right)=\sum_{k=1, k \text { odd }}^{n} 2\left(1+c_{k} \cos k t\right) .
$$

Now, let $A$ be the set of indices $k$ for which $c_{k}=1$ and $B$ the set of indices $k$ for which $c_{k}=-1$. Then

$$
\begin{aligned}
1+c_{k} \cos k t & = \begin{cases}1+\cos k t & \text { if } k \in A, \\
1-\cos k t & \text { if } k \in B,\end{cases} \\
& = \begin{cases}2 \cos ^{2}(k t / 2) & \text { if } k \in A, \\
2 \sin ^{2}(k t / 2) & \text { if } k \in B .\end{cases}
\end{aligned}
$$

Then

$$
P\left(e^{i t}\right)=\sum_{k \in A} 4 \cos ^{2}(k t / 2)+\sum_{k \in B} 4 \sin ^{2}(k t / 2)
$$


Since every summand on the right-hand side of this identity is nonnegative, in view of the AM-GM inequality, we obtain

$$
P\left(e^{i t}\right) \geqslant \frac{n+1}{2}\left(\prod_{k \in A} 4 \cos ^{2}(k t / 2) \prod_{k \in B} 4 \sin ^{2}(k t / 2)\right)^{\frac{2}{n+1}} .
$$

Note that there may be at most finitely many points $t \in[0,2 \pi]$, where the expressions on both sides of the last inequality vanish. For all the other $t$, we can take the logarithm of both sides and get

$$
\log \left|P\left(e^{i t}\right)\right| \geqslant \log \frac{n+1}{2}+\frac{2}{n+1}\left(\sum_{k \in A} \log 4 \cos ^{2}(k t / 2)+\sum_{k \in B} \log 4 \sin ^{2}(k t / 2)\right) .
$$

The summands on both sides now are integrable for $t \in[0,2 \pi]$. By Lemma 3.1, the integrals of $\log 4 \cos ^{2}(k t / 2)$ and $\log 4 \sin ^{2}(k t / 2)$ vanish. Thus we have

$$
\log M(P)>\log \frac{n+1}{2}
$$

by (2.1). Observe that the inequality must be strict, since the AM-GM equality can hold at most on a finite number of points $e^{i t}$. This completes the proof.

Proof of Theorem 2.6. It suffices to consider the "+" case in $R_{n}$, since the "-" case is $R_{n}(-z)$. We may also assume $n \geq 3$. Let $z:=e^{i t}$. Then

$$
\begin{aligned}
R_{n}(z) & =(n+1)+\sum_{k=-n, k \text { odd }}^{n} z^{k} \\
& =(n+1)+\frac{z^{n+1}-\bar{z}^{(n+1)}}{z-\bar{z}} \\
& =\frac{(n+1) \sin t+\sin (n+1) t}{\sin t} .
\end{aligned}
$$

Now using Lemma 3.2 with $a=n+1, b=1, x=t$ and $\alpha=n t$, we have

with

$$
R_{n}\left(e^{i t}\right)=c(n, t) \frac{\sin (t+\beta(n, t))}{\sin t}
$$

$c(n, t)=\sqrt{(n+1)^{2}+1+2(n+1) \cos (n t)}, \quad \beta(t)=\tan ^{-1}\left(\frac{\sin (n t)}{n+1+\cos (n t)}\right)$, since $a+b \cos \alpha=(n+1)+\cos n t \geqslant n>0$. Observe that

$$
n \leqslant c(n, t) \leqslant n+2
$$

and

$$
|\beta(t)| \leq \tan ^{-1}\left(\frac{1}{n}\right)<\frac{1}{n} .
$$

The last inequality follows from $\tan ^{-1}|t| \leq|t|$ for all $t \in \mathbb{R}$. By Jensen's formula, we have

$$
\begin{aligned}
2 \pi \log M\left(R_{n}\right)-\int_{0}^{2 \pi} \log c(n, t) d t & =\int_{0}^{2 \pi}\left(\log \left|R_{n}\left(e^{i t}\right)\right|-\log c(n, t)\right) d t \\
& =\int_{0}^{2 \pi} \log |\sin (t+\beta(n, t))|-\log |\sin t| d t .
\end{aligned}
$$


We now estimate the integral of $\log |\sin (t+\beta(n, t))|$ by using Lemma 3.4. Let

$$
I=\int_{0}^{2 \pi} \log |\sin (t+\beta(n, t))| d t=\int_{-1 / n}^{2 \pi-1 / n} \log |\sin (t+\beta(n, t))| d t
$$

because the integral is periodic with period $2 \pi$. We now write

$$
I=\int_{I(0) \cup I(\pi / 2) \cup I(\pi) \cup I(3 \pi / 2)} \log |\sin (t+\beta(n, t))| d t+\int_{J} \log |\sin (t+\beta(n, t))| d t,
$$

where $I(a)=[a-1 / n, a+1 / n]$ as in Lemma 3.3 and $J$ is the complement of the disjoint union $I(0) \cup I(\pi / 2) \cup I(\pi) \cup I(3 \pi / 2)$ in $[-1 / n, 2 \pi-1 / n)$.

In view of Lemma 3.4, we first have

$$
\int_{I(0) \cup I(\pi / 2) \cup I(\pi) \cup I(3 \pi / 2)} \log |\sin (t+\beta(n, t))| d t=-\frac{4}{n} \log n+O\left(\frac{1}{n}\right) .
$$

Since the function $|\sin t|$ is increasing for $t \in[0, \pi / 2]$ and $t \in[\pi, 3 \pi / 2]$, we find

$$
\begin{aligned}
\int_{J \cap([0, \pi / 2] \cup[\pi, 3 \pi / 2])} \log |\sin (t+\beta(n, t))| d t & \geq \int_{J \cap([0, \pi / 2] \cup[\pi, 3 \pi / 2])} \log |\sin (t-1 / n)| d t \\
& =\left(\int_{0}^{\pi / 2-2 / n}+\int_{\pi}^{3 \pi / 2-2 / n}\right) \log |\sin t| d t .
\end{aligned}
$$

Similarly, since the function $|\sin t|$ is decreasing in intervals $[\pi / 2, \pi]$ and $[3 \pi / 2,2 \pi]$, we find

$$
\begin{aligned}
\int_{J \cap([\pi / 2, \pi] \cup[3 \pi / 2,2 \pi])} \log |\sin (t+\beta(n, t))| d t & \geq \int_{J \cap([\pi / 2, \pi] \cup[3 \pi / 2,2 \pi])} \log |\sin (t+1 / n)| d t \\
& =\left(\int_{\pi / 2+2 / n}^{\pi}+\int_{3 \pi / 2+2 / n}^{2 \pi}\right) \log |\sin t| d t .
\end{aligned}
$$

Therefore, we have

$$
\begin{aligned}
\int_{J} \log |\sin (t+\beta(n, t))| d t & \geq \int_{0}^{2 \pi} \log |\sin t| d t-\left(\int_{\pi / 2-2 / n}^{\pi / 2+2 / n}+\int_{3 \pi / 2-2 / n}^{3 \pi / 2+2 / n}\right) \log |\sin t| d t \\
(4.6) & =\int_{0}^{2 \pi} \log |\sin t| d t+O\left(\frac{1}{n}\right)
\end{aligned}
$$

by Lemma 3.4. In view of (4.4), (4.5) and (4.6), we have

$$
\int_{0}^{2 \pi} \log |\sin (t+\beta(n, t))| d t \geq \int_{0}^{2 \pi} \log |\sin t| d t-\frac{4}{n} \log n+O\left(\frac{1}{n}\right) .
$$

Hence from (4.3),

$$
2 \pi \log M\left(R_{n}\right)>\int_{0}^{2 \pi} \log c(n, t) d t-\frac{4}{n} \log n+O\left(\frac{1}{n}\right) .
$$


Finally, since

$$
c(n, t)=\sqrt{(n+1)^{2}+1+(2 n+1) \cos t}=n \sqrt{1+O(1 / n)},
$$

so $\log c(n, t)=\log n+O(1 / n)$. Hence

$$
\log M\left(R_{n}\right)>\log n-\frac{2}{n \pi} \log n+O\left(\frac{1}{n}\right) .
$$

Applying the exponent on both sides of the above inequality and using $e^{-t}>1-t$ for $t>0$ one obtains

$$
M\left(R_{n}\right)>n\left(1-\frac{2}{n \pi} \log n+O\left(\frac{1}{n}\right)\right)=n-\frac{2}{\pi} \log n+O(1),
$$

as claimed.

\section{REFERENCES}

[1] R.H. BARKer, Group synchronizing of binary digital systems, Communication theory, 273287, Butterworths Sci. Pub., London, 1953.

[2] P. Borwein, E. Kaltofen, M.J. Mossinghoff, Irreducible polynomials and Barker sequences, ACM Commun. Comput. Algebra 41 (2007), no. 3-4, 118-121. MR2404490 (2009b:94035)

[3] P. Borwein, M.J. Mossinghoff, Number theory and polynomials, 71-88, London Math. Soc. Lecture Note Ser., 352, Cambridge Univ. Press, Cambridge, 2008. MR2428516 (2009k:11040)

[4] S. Eliahou, M. Kervaire, Barker sequences and difference sets, Enseign. Math. (2) 38 (1992), no. 3-4, 345-382. MR.1189012 (93i:11018)

[5] S. Eliahou, M. Kervaire, B. Saffari, A new restriction on the lengths of Golay complementary sequences, J. Combin. Theory Ser. A 55 (1990), no. 1, 49-59. MR1070014 (91i:11020)

[6] P. Fan, M. Darnell, Sequence design for communications applications, Research Studies Press, Somerset, England, 1996.

[7] J. JEDWAB, A survey of the merit factor problem for binary sequences, Sequences and Their Applications, Proceedings of SETA 2004, Lecture Notes in Computer Science 3486, 30-55, Springer Verlag, Berlin, 2005.

[8] J. Jedwab, S. Lloyd, A note on the nonexistence of Barker sequences, Des. Codes Cryptogr. 2 (1992), no. 1, 93-97. MR.1157481 (93e:11032)

[9] M.J. Mossinghoff, Wieferich Pairs and Barker Sequences, Des. Codes Cryptogr. 53 (2009), no. 3, 149-163. MR2545689 (2011c:11039)

[10] D.J. Newman, Norms of polynomials, Amer. Math. Monthly 67 (1960), 778-779. MR0125205 (23:A2510)

[11] D.J. Newman, An $L_{1}$ extremal problem for polynomials, Proc. Amer. Math. Soc. 16 (1965), 1287-1290. MR0185119 (32:2589)

[12] K.H. Leung, B. Schmidt, The field descent method, Des. Codes Cryptogr. 36 (2005), no. 2, 171-188. MR2211106 (2007g:05023)

[13] B. SAFFARI, Barker sequences and Littlewood two-sided conjectures on polynomials with \pm 1 coefficients, Séminaire d'Analyse Harmonique, Année 1989/90, 139-151, Univ. Paris XI, Orsay, 1990. MR:1104693 (92i:11032)

[14] C. Sмүтн, The Mahler measure of algebraic numbers: a survey, Number theory and polynomials, 322-349, London Math. Soc. Lecture Note Ser., 352, Cambridge Univ. Press, Cambridge, 2008. MR2428530 (2009j:11172)

[15] R. TuRYN, On Barker codes of even length, IEEE Trans. Inform. Theory 51 (1963), no. 9, 1256.

[16] R. Turyn, Character sums and difference sets, Pacific J. Math. 15 (1965), 319-346. MR0179098 (31:3349)

[17] R. TuRYN, Sequences with small correlation, Error Correcting Codes (Proc. Sympos. Math. Res. Center, Madison, Wis.), 195-228, John Wiley, New York, 1968. MR0242566 (39:3897)

[18] R. Turyn, J. Storer, On binary sequences, Proc. Amer. Math. Soc. 12 (1961), 394-399. MR0125026 (23:A2333) 
[19] J.E. Littlewood, On polynomials $\sum^{n} \pm z^{m}, \sum^{n} e^{\alpha_{m} i} z^{m}, z=e^{\Theta i}$, J. London Math. Soc. 41 (1966), 367-376. MR0196043 (33:4237)

[20] J.E. LitTlewood, Some problems in real and complex analysis, D.C. Heath and Co., Lexington, Mass., 1968. MR0244463 (39:5777)

Department of Mathematics, Simon Fraser University, 8888 University Drive, Burnaby, British Columbia V5A 1S6, Canada

E-mail address: pborwein@sfu.ca

Department of Mathematics, Simon Fraser University, 8888 University Drive, Burnaby, British Columbia V5A 1S6, Canada

E-mail address: kkchoi@math.sfu.ca

Department of Mathematics and Informatics, Vilnius University, Naugarduko 24, Vilnius LT-03225, Lithuania

E-mail address: jonas.jankauskas@gmail.com 